\title{
Early-Onset Vascular Dementia in a 43-Year-Old Man with Accelerated Atherosclerotic Disease, Elevated Lipoprotein (a), and a Missense DNAJC5 Variant with Potential Association to Adult-Onset Ceroid Lipofuscinosis
}

\author{
Ridwan Naim Faruq ${ }^{a}$ Prajna D'Silva ${ }^{a}$ Freddy Duarte Lau $^{a}$ Chen Zhao ${ }^{b}$ \\ Sachin Majumdara, c \\ aDepartment of Internal Medicine, Bridgeport Hospital, Yale New Haven Health System, \\ Bridgeport, CT, USA; 'bepartment of Genetics, Yale University School of Medicine, New \\ Haven, CT, USA; 'Section of Endocrinology, Bridgeport Hospital, Yale New Haven Health \\ System, Bridgeport, CT, USA
}

\section{Keywords}

Dementia $\cdot$ Lipoprotein (a) $\cdot$ Ceroid lipofuscinosis

\begin{abstract}
Early-onset dementia is defined as dementia occurring prior to the age of 65 . Given its impact on physical, mental, and socioeconomic well-being, it is crucial to identify modifiable risk factors. Here, we report a 43-year-old man with early-onset dementia associated with elevated lipoprotein (a) and a missense variant in the DNAJC5 gene. He presented to the hospital with memory loss and multiple cerebrovascular infarcts. Eight months prior, an MRI revealed small acute and subacute infarcts involving the left PCA for which he was treated with antiplatelet agents and a statin. Three months later, he was readmitted for progressive memory loss. CT imaging showed evolving and new infarcts compared to prior scans. A cardiac echocardiogram excluded thrombus and PFO, and he was diagnosed with early vascular dementia. He was readmitted again 5 months later with additional evaluation revealing multifocal moderate to severe stenosis and irregularities involving the bilateral ICA and bilateral PCAs. MRI showed more pronounced infarcts compared to a previous MRI as well as new infarcts. CSF studies, VDRL, RF, ANA, ANCA, homocysteine, and MMA levels were normal. Lipoprotein (a) was found to be markedly elevated, and genetic testing revealed a missense variant of the DNAJC5 gene, the mutation of which is associated with ceroid lipofuscinosis. In conclusion, in patients with
\end{abstract}


early-onset dementia and evidence of accelerated atherosclerosis, it is reasonable to measure $\mathrm{Lp}(\mathrm{a})$ and consider testing for variants in genes such as DNAJC5 and others, particularly when disease severity appears unexplained by known risk factors or circumstances.

(C) 2021 The Author(s).

Published by S. Karger AG, Basel

\section{Introduction}

Dementia is a syndrome of chronic, progressive cognitive decline in domains including memory, orientation, comprehension, and calculation beyond what is expected of normal aging. Vascular dementia is the most common cause of dementia after Alzheimer's dementia, and its cause appears multifactorial arising from vascular components of atherosclerosis, arteriosclerosis, microinfarcts, and amyloid angiopathy, in association with neurodegeneration [1]. Gorelick [2] broadly categorized the risk factors for vascular dementia into 4 major categories: demographic (male and age), atherosclerotic (hypertension, cigarette smoking, MI, DM, and hyperlipidemia), genetic (CADASIL and possibly apolipoprotien E), and stroke related.

The incidence of vascular dementia rises with age with exponential increases occurring between the ages of 65 and 90, essentially doubling every 5 years [3]. However, there is a paucity of information on early-onset dementia (dementia with onset before age 65). A cohort study conducted in subjects with a mean initial age of 18 years, who were followed for a median of 37 years, identified 9 independent risk factors for early-onset dementia including alcohol and other drug use, stroke, high blood pressure, and low overall cognitive function [4]. The most common causes were still Alzheimer's dementia (22.3\%) and vascular dementia (15.9\%). Unfortunately, due to the lower incidence of early-onset dementia, it is infrequently recognized and managed on time. One study found that the duration between symptom onset and diagnosis of early-onset dementia was 4.4 years compared to 2.8 years for late-onset dementia [5]. Therefore, it is crucial to identify significant risk factors that could contribute to early-onset dementia so that a diagnosis can be made and a timely intervention could be initiated to prevent further progression. Herein, we report a case of early-onset vascular dementia in a young man with potential risk factors, elevated lipoprotein (a) and a missense variant in the DNAJC5 gene, associated with ceroid lipofuscinosis.

\section{Case Report}

A 43-year-old man presented to the hospital with a history of progressive memory loss. He would get lost frequently, had trouble recalling recent events, and had been sent home from his job on several occasions due to the inability to perform his duties. Eight months prior to his presentation, he had been admitted to the hospital for a hyperosmolar hyperglycemic state and found to have a small subacute infarct involving the left posterior cerebral artery distribution. He was started on $80 \mathrm{mg}$ of atorvastatin and dual antiplatelet therapy with aspirin and clopidogrel as well as insulin for diabetes. He was discharged home, yet soon after, his fiancé started noticing further progression of memory loss, and he was readmitted to the hospital for further evaluation. CT imaging showed an evolving acute to subacute right occipital lobe infarct with an acute to subacute left anterior thalamic lacunar infarct, which were new compared to prior scan. Given that his infarcts were in multiple territories, an echocardiogram was done which ruled out thrombus. He was diagnosed with early vascular

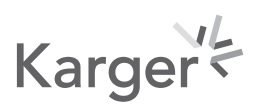




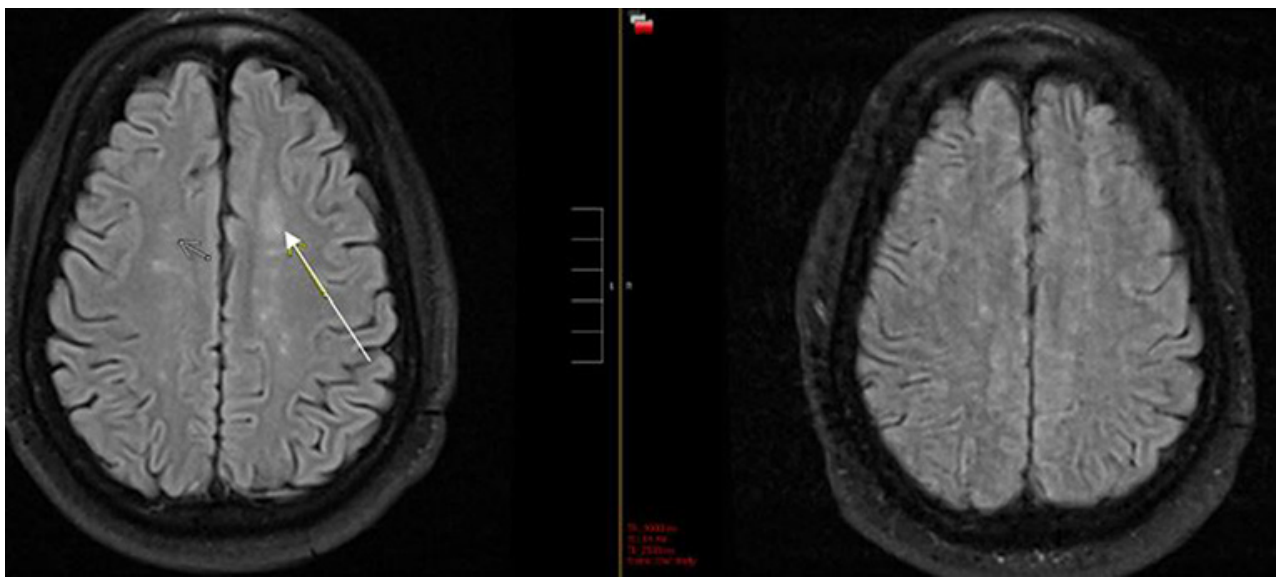

Fig. 1. New infarct (arrow in the left image) in recent MRI compared to previous MRI (right image).

dementia and continued on medical therapy for aggressive risk factor modification. He was discharged home to the care of his mother-in-law and fiancé for approximately 5 months. However, due to difficulties in taking care of him by his fiancé and mother-in-law, he was referred for a third admission to better define his condition and needs while the court assigned a conservator for him as psychiatric evaluation deemed him to lack capacity for medical decision-making.

His past medical history was significant for heart failure, diabetes mellitus, hypertension, hyperlipidemia, obesity, and obstructive sleep apnea. There was no history of early stroke or dementia in his parents, and he has a healthy sister without any known medical conditions. Examination revealed BP: 168/91, HR: 110, and RR: 18. Initial neurologic exam showed correct orientation to place and person and a GCS of 15 . However, he was unable to state the current month, subtract serial $7 \mathrm{~s}$ without prompting, or to tell the months of the year backwards, yet no aphasia or neglect was noted. He also failed the 3-word recall test.

CTA of the head showed multifocal moderate to severe stenosis and irregularities including bilateral distal ICA (moderate) R M1 (mild to moderate) and nearly occluded bilateral PCAs. MRI showed bilateral subcortical infarcts (as shown by a white arrow in Fig. 1) which were more pronounced compared to a previous MRI 8 months ago. There was also a new subacute posterior corpus callosum hyperintensity (as shown by a white arrow in Fig. 2) and a right cortical occipital and left pontine stroke, all of which were not seen on previous MRI. The patient was believed to have accelerated intracranial atherosclerosis, but given his age and the dramatic clinical and MRI progression over the course of only 8 months, alternative diagnoses such as vasculitis, intravascular lymphoma, sarcoidosis, and infectious etiologies were considered. Cerebrospinal fluid (CSF) studies showed clear fluid with a white cell count of $5 / \mu \mathrm{L}$, red cell count of $2,000 / \mu \mathrm{L}$, glucose of $74 \mathrm{mg} / \mathrm{dL}$, and protein of $65.4 \mathrm{mg} / \mathrm{dL}$. CSF VDRL was negative. ESR was $24 \mathrm{~mm} / \mathrm{h}$, and hsCRP was $4.4 \mathrm{mg} / \mathrm{L}$. RF, ANA, and ANCA were negative. ACE level was $<5$ (units). His lipid panels before and after initiation of $80 \mathrm{mg}$ of daily atorvastatin revealed a total cholesterol of 219 and 101, HDL 36 and 29, and LDL 160 and 63, in milligrams per deciliter, respectively. Homocysteine and MMA levels were normal. Lipoprotein little a (Lpa) was checked on 2 occasions while on statin therapy when his total cholesterol levels were 101 and $107 \mathrm{mg} / \mathrm{dL}$ and was found to be 267 and $287 \mathrm{~nm}$ ( $<75 \mathrm{nM}$ ). Ezetimibe had been added at a dose of $10 \mathrm{mg}$ a day in between measures of Lp(a).

Genetic testing was performed to look for variants in $\mathrm{Lp}(\mathrm{a})$ and other genes associated with early-onset dementia and revealed a heterogeneous variant in DNAJC5 NM_025219:exon3:c.C188T:p.A63V (chr20:62560745 [hg19]), a gene commonly associated

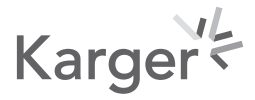




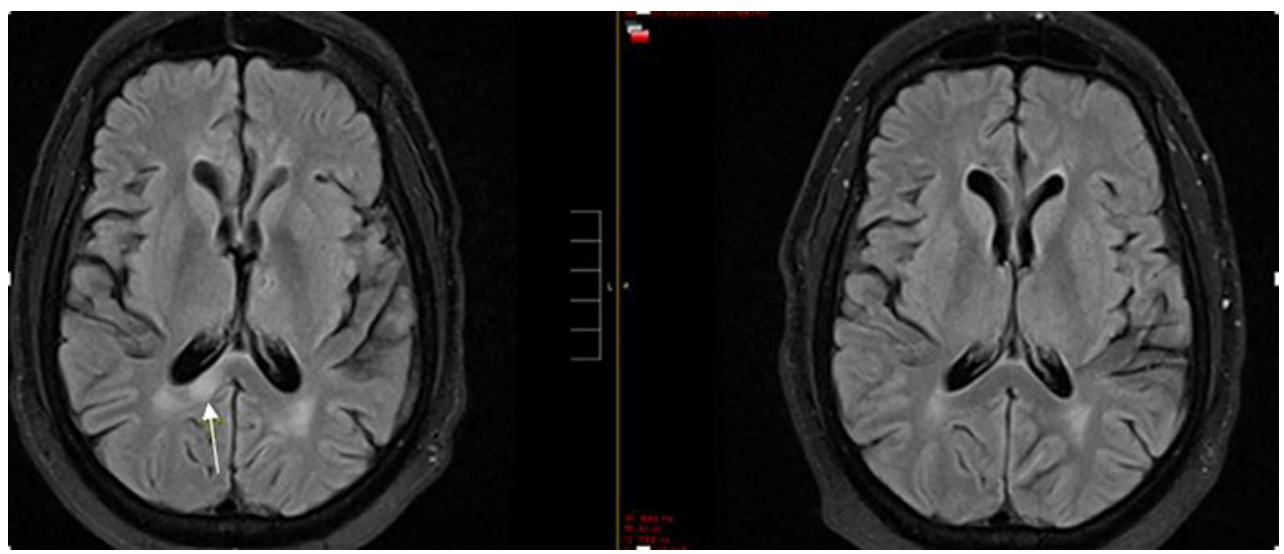

Fig. 2. New infarct (arrow in the left image) in recent MRI compared to previous MRI (image in right).

with ceroid lipofuscinosis (neuronal, 4, parry type). The variant was identified using exome sequencing where paired end sequence reads were converted to FASTQ format and were aligned to the reference human genome assembly GRCh37/hg19. Following variant annotation using a Genome Analysis Toolkit (GATK) and AnnoVar, filtering was applied against allele frequencies and disease citations using databases including the Genome Aggregation Database, an internal database of Yale DNA Diagnostics Laboratory, ClinVAR, OMIM, and other in silico predictors of functional consequences. Variants with allele frequency above $3 \%$ were excluded from further analysis. Genes of variants with an allele frequency $<3 \%$ were cross-compared with panel(s) of lab-curated genes associated with condition(s) including cerebrovascular disease, hyperlipidemia, and dementia that can explain the patient's phenotype (refer to the gene list in online suppl. Section; for all online suppl. material, see www.karger.com/doi/10.1159/000518194).

\section{Discussion}

Lipoprotein(a) (Lp[a]) is an LDL particle in which the apo (a) component shares a homology with plasminogen and can inhibit fibrinolysis; it has the potential to promote vascular thrombosis and has been identified as an independent risk factor for cardiovascular disease in several large epidemiologic studies [6]. Additionally, a number of studies indicate that it is a risk factor for stroke, and a recent mendelian randomization study has provided evidence for a causal role of $L p(a)$ in large artery stroke; interestingly, significantly increased Lp(a) levels in stroke appear to correlate with large, rather than small, vessel disease [7]. A number of studies also show a link between higher $\operatorname{Lp}(\mathrm{a})$ concentrations and vascular dementia (VD) thus extending the role of $L p(a)$ in promoting cardio and cerebrovascular disease, ischemia, and inflammation, to cognitive decline [8]. Thus, high serum levels of Lp(a) could be considered a clinical hallmark to distinguish VD from Alzheimer's. Our patient presented with strokes and diffuse vascular disease in the setting of markedly elevated Lp(a) levels. While elevations in $\mathrm{Lp}(\mathrm{a})$ in conjunction with typical risk factors such as HTN and poorly controlled diabetes may explain his presentation, we additionally identified a missense variant in the DNAJC5 gene which raises the possibility of an additional contributor to his early-onset dementia. In silico tools (SIFT: deleterious; PolyPhen-2: probably damaging) predict a likely deleterious effect to the protein structure and/or activity caused by this variant.

\section{Karger'}


The DNAJC5 gene codes for human cysteine string protein, a member of the DnaJ/Hsp40 family of co-chaperones that localizes to neuronal synaptic vesicles and contributes to the maintenance of protein folding in the presynaptic compartment [9]. Recent studies have revealed that the variants in the DNAJC5 gene are responsible for the neurodegenerative disease, adult-onset neuronal ceroid lipofuscinosis (ANCL) [9]. ANCL also known as autosomal dominant Kufs' disease and Parry disease is an extremely rare hereditary neurodegenerative disorder with a highly variable presentation including generalized epilepsy, movement disorders, and early-onset dementia [9]. The disease has a mean age of onset of 30 years and progresses rapidly upon diagnosis, with death occurring on average at 45 years of age [9]. The variant of the DNAJC5 gene detected in our patient has not been previously associated with ceroid lipofuscinosis. However, because of the early-onset dementia exhibited by our patient, we cannot exclude the possibility that the variant detected in our patient may also be linked to ANCL.

The variant detected in our patient is present in the public variant database such as gnomAD (gnomAD, $0.004 \%$ ), and it has been recently curated as a variant of uncertain significance in ClinVar (variation ID: 558992). There could be few explanations to the presence of this variant in public databases. First, DNAJC5 is associated with an adult-onset condition (ceroid lipofuscinosis), whereas affected individuals at their young age without disease manifestation could be selected and included as testing population in the gnomAD database. Indeed, very limited studies were done to thoroughly examine symptoms of individuals carrying the disease-causing DNAJC5 variant at their young age. Second, it is known that certain ethnic groups can carry the disease-causing variant at much higher allele frequency than in the general population (founder effect). Indeed, this variant displays an unequal distribution with the highest allele frequency $(0.01 \%)$ reported from non-Finnish European population in the gnomAD variant database. With limited family history, it is uncertain whether higher than expected allele frequency of this variant can be partially explained by a potential stratified testing population. Third, this variant has higher incidence compared to that of ANCL which could possibly be explained by nonpenetrance of this variant in causing ANCL. However, there are no data regarding degree of penetrance of this variant in ANCL. Given the presence of this variant in public databases, we cannot exclude the rare possibility that this variant is coincidental, and it may not be associated with the vascular dementia in this patient. Although variants in DNAJC5 have been found in families with ANCL showing autosomal dominant inheritance, sporadic cases of ANCL have also been linked to DNAJC5 variants [10]. We suspect it could be a sporadic variant in our patient given the absence of early-onset dementia in his immediate family members. Unfortunately, his family members declined genetic testing, so we were unable to determine their genotypes.

Although the manifestations of ANCL associated with the DNAJC5 gene are highly variable, there are currently no data linking DNAJC5 variants to accelerated atherosclerosis as a mechanism for dementia or any potential interaction with $\operatorname{Lp}(\mathrm{a})$. However, in previous cases of ANCL reported, lipoprotein (a) was not measured, and therefore we cannot determine if a correlation exists. Given the overlap of accelerated atherosclerosis and early-onset dementia in our case, we cannot exclude a relationship between elevated Lp(a) and DNAJC5 variantrelated disease. Therefore, in patients with early-onset dementia with evidence of accelerated atherosclerosis, it would be helpful to measure $\mathrm{Lp}(\mathrm{a})$ and perform genetic testing to look for DNAJC5 or other gene variants which may play a role in dementia, either independently or synergistically.

His elevated $\operatorname{Lp}(\mathrm{a})$, a clear risk factor for further vascular events, did not respond to standard lipid-lowering therapy. Despite its clinical significance, reduction of Lp(a) can be challenging. Lifestyle interventions such as diet and exercise have proven ineffective, and no

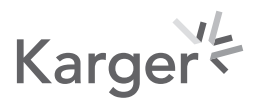


current pharmacotherapies have been approved for this purpose. No available data have been generated to suggest improved clinical outcomes, including stroke, with treatment of an elevated Lp(a) level.

Statins have not been shown to effectively lower Lp(a) and may, according to some studies, raise $\mathrm{Lp}(\mathrm{a})$. A meta-analysis of 5,256 patients showed positive mean $\mathrm{Lp}(\mathrm{a})$ changes in individuals taking statins versus placebo [11] while another meta-analysis of 29,069 patients demonstrated a linear association between Lp(a) levels $\geq 50 \mathrm{mg} / \mathrm{dL}$ and CV events for individuals on statin drugs, a risk that was more pronounced at younger ages [12].

Contemporary studies on PCSK9 inhibitors have shown that these drugs are capable of reducing Lp(a) by a mean of 15-25\% either alone or in combination with statin therapy [13]. Both ODYSSEY and FOURIER trials revealed significant relative risk reduction of CV events with $\mathrm{Lp}(\mathrm{a})$ lowering in patients treated with alirocumab and evolocumab versus placebo, respectively [13] A recent multicenter international phase $2 \mathrm{~b}$ trial that included 286 patients with pre-existing CVD and baseline $\mathrm{Lp}(\mathrm{a})>60 \mathrm{mg} / \mathrm{dL}$ demonstrated a dose-dependent $\mathrm{Lp}(\mathrm{a})$ reduction in all groups treated with IONIS-APO(a) Lrx [14].

Lipoprotein apheresis is considered a last-line therapy [15]. While highly effective in reducing $\mathrm{Lp}(\mathrm{a})$ levels, it is time-consuming and expensive and thus rarely used. Whether significant $\mathrm{Lp}(\mathrm{a})$ reduction can provide a protective effect in the development or progression of ischemic stroke requires more dedicated studies.

\section{Conclusion}

We report a young man with early-onset dementia associated with elevated Lp(a) and a missense variant in the DNAJC5 gene. To our knowledge, there is no known association between DNAJC5 variants and Lp(a) or vascular dementia, rather its primary association is with adult-onset ceroid lipofuscinosis. While our patient's features can be explained by elevated $\mathrm{Lp}(\mathrm{a})$ in the setting of established risk factors, one cannot exclude a role for this DNAJC5 variant in his presentation. Therefore, in patients with early-onset dementia accompanied by accelerated atherosclerosis, it would be important to include $\mathrm{Lp}(\mathrm{a})$ in the workup and consider further evaluation to identify genetic risk factors. At present, the use of PCSK9 inhibitors might be the best treatment option for reducing Lp(a) levels.

\section{Statement of Ethics}

Written informed consent was obtained from the legal conservator of the patient for publication of this case report and any accompanying images. This study is exempt from ethics committee approval as no ethical issues arose from reporting this case.

\section{Conflict of Interest Statement}

The authors have no conflicts of interest to declare.

\section{Funding Sources}

The authors received no funding.

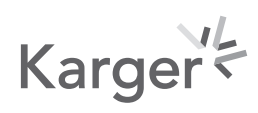




\section{Author Contributions}

Ridwan Naim Faruq was involved in clinical evaluation, drafting the case report, and discussion. Prajna D'Silva was involved in patient follow-up and drafting introduction. Freddy Lau Duarte was involved in patient care and drafting discussion. Chen Zhao was involved in genetic testing, drafting of methods, and discussion. Sachin Majumdar was involved in clinical evaluation and manuscript revision.

\section{Data Availability Statement}

All data generated or analyzed during this study are included in this article and its online suppl. files. Further enquiries can be directed to the corresponding author.

\section{References}

1 Wolters FJ, Ikram MA. Epidemiology of vascular dementia. Arterioscler Thromb Vasc Biol. 2019 Aug 1;39(8): 1542-9.

2 Gorelick PB. Risk factors for vascular dementia and Alzheimer disease. Stroke. 2004 Nov 1;35(11 Suppl 1): 2620-2.

3 Wolters FJ, Ikram MA. Epidemiology of dementia: the burden on society, the challenges for research. Methods Mol Biol. 2018;1750:3-14.

4 Nordström P, Nordström A, Eriksson M, Wahlund L-O, Gustafson Y. Risk factors in late adolescence for youngonset dementia in men: a nationwide cohort study. JAMA Intern Med. 2013;173(17):1612-8.

5 van Vliet D, de Vugt ME, Bakker C, Pijnenburg YA, Vernooij-Dassen MJ, Koopmans RT, et al. Time to diagnosis in young-onset dementia as compared with late-onset dementia. Psychol Med. 2013 Feb;43(2):423-32.

6 Berg K, Dahlén G, Christophersen B, Cook T, Kjekshus J, Pedersen T. Lp(a) lipoprotein level predicts survival and major coronary events in the scandinavian simvastatin survival study. Clin Genet. 1997 Nov;52(5):25461.

7 Pan Y, Li H, Wang Y, Meng X, Wang Y. Causal effect of Lp(a) [lipoprotein(a)] level on ischemic stroke and Alzheimer disease: a mendelian randomization study. Stroke. 2019 Dec;50(12):3532-9.

8 Spada RS, Ferri R, Roccasalva G, Toscano G, Cosentino FI. Lipoprotein(a), vascular dementia and Alzheimer's disease. Arch Gerontol Geriatr Suppl. 2001 Feb 1;7:369-73.

9 Burgoyne RD, Morgan A. Cysteine string protein (CSP) and its role in preventing neurodegeneration. Semin Cell Dev Biol. 2015 Apr;40:153-9.

10 Naseri N, Sharma M, Velinov M. Autosomal dominant neuronal ceroid lipofuscinosis: clinical features and molecular basis. Clin Genet. 2021;99(1):111-8.

11 Tsimikas S, Gordts PLSM, Nora C, Yeang C, Witztum JL. Statin therapy increases lipoprotein(a) levels. Eur Heart J. 2020 Jun 21;41(24):2275-84.

12 Willeit P, Ridker PM, Nestel PJ, Simes J, Tonkin AM, Pedersen TR, et al. Baseline and on-statin treatment lipoprotein(a) levels for prediction of cardiovascular events: individual patient-data meta-analysis of statin outcome trials. Lancet. 2018 Oct 13;392(10155):1311-20.

13 Bittner VA, Szarek M, Aylward PE, Bhatt DL, Diaz R, Edelberg JM, et al. Effect of alirocumab on lipoprotein(a) and cardiovascular risk after acute coronary syndrome. J Am Coll Cardiol. 2020 Jan 21;75(2):133-44.

14 Tsimikas S, Karwatowska-Prokopczuk E, Gouni-Berthold I, Tardif JC, Baum SJ, Steinhagen-Thiessen E, et al. Lipoprotein(a) reduction in persons with cardiovascular disease. N Engl J Med. 2020 Jan 16;382(3):244-55.

15 Cuchel M, Bruckert E, Ginsberg HN, Raal FJ, Santos RD, Hegele RA, et al. Homozygous familial hypercholesterolaemia: new insights and guidance for clinicians to improve detection and clinical management. A position paper from the consensus panel on familial hypercholesterolaemia of the European Atherosclerosis Society. Eur Heart J. 2014 Aug 21;35(32):2146-57. 